\title{
SIMPLE DERIVATIONS OF PROPERTIES OF COUNTING PROCESSES ASSOCIATED WITH MARKOV RENEWAL PROCESSES
}

\author{
FRANK BALL, ${ }^{*}$ The University of Nottingham \\ ROBIN K. MILNE, ${ }^{* *}$ The University of Western Australia
}

\begin{abstract}
A simple, widely applicable method is described for determining factorial moments of $\hat{N}_{t}$, the number of occurrences in $(0, t]$ of some event defined in terms of an underlying Markov renewal process, and asymptotic expressions for these moments as $t \rightarrow \infty$. The factorial moment formulae combine to yield an expression for the probability generating function of $\hat{N}_{t}$, and thereby further properties of such counts. The method is developed by considering counting processes associated with events that are determined by the states at two successive renewals of a Markov renewal process, for which it both simplifies and generalises existing results. More explicit results are given in the case of an underlying continuous-time Markov chain. The method is used to provide novel, probabilistically illuminating solutions to some problems arising in the stochastic modelling of ion channels.
\end{abstract}

Keywords: Continuous-time Markov chain; counting process; factorial moment; factorial moment measure; ion channel modelling; Markov renewal process; point process; semiMarkov process; time interval omission

2000 Mathematics Subject Classification: Primary 60K15

Secondary 60G55; 60J27; 60K20

\section{Introduction}

Several works, for example Darroch and Morris (1967), Ball and Sansom (1987), Masuda and Sumita (1987), Csenki (1991), (1994, especially Chapters 3, 5, 9, 10, and 11), (1995), Ball et al. (1993), Ball and Davies (1997), Ball (1997), and Zheng (2001), have dealt with properties of particular counting processes defined on an underlying continuous-time Markov chain or Markov renewal process with a finite state space. In almost all cases, an important aspect was the derivation of an expression for the probability generating function (or equivalent transform), and hence the (first few) moments, of a random variable $\hat{N}_{t}$ counting the number of occurrences in the time interval $(0, t]$ of some event defined in terms of the underlying process, for example the number of visits during $(0, t]$ to a specified subset of the state space. In many cases the derivations were lengthy, involving, for example, forward or backward equations. Moreover, the formulae obtained for the moments were often difficult, if indeed possible, to evaluate in practice, so asymptotic expressions as $t \rightarrow \infty$ were derived from corresponding Laplace transforms using a Tauberian argument.

Received 11 December 2002; revision received 28 June 2005.

* Postal address: School of Mathematical Sciences, The University of Nottingham, Nottingham NG7 2RD, UK.

Email address: frank.ball@ nottingham.ac.uk

** Postal address: School of Mathematics and Statistics, The University of Western Australia, Crawley, WA 6009, Australia. Email address: milne@maths.uwa.edu.au 
The purpose of this paper is to present a unified and simpler approach to problems of the above type. For many counting processes defined on a Markov renewal process, the event times together with the corresponding state of the underlying process form a derived Markov renewal process that is embedded within the underlying Markov renewal process; see, for example, the treatment by Çinlar (1969) of counters, and those by Ball and Sansom (1987) and Masuda and Sumita (1987) of the number of visits to a subset of the state space. Properties of $\hat{N}_{t}$ then follow because $\hat{N}_{t}$ is just the total number of renewals in the derived process. In this paper, we adopt a different approach. We view the counts $\hat{N}_{t}, t \geq 0$, as a point process and use the classical result expressing factorial moments of a point process as integrals of factorial moment densities. For many counting processes the latter can be obtained very easily by exploiting conditional independence properties of the underlying Markov renewal process, leading to expressions for the factorial moments of $\hat{N}_{t}$ in terms of the Markov renewal kernel density of the underlying Markov renewal process. This, in turn, leads to an expression for the probability generating function of $\hat{N}_{t}$, from which other properties related to such counts (for example, those of waiting times to the $k$ th occurrence of the event under consideration) can be obtained.

The approach used in this paper has several advantages over previous methods. Firstly, it is simpler and more direct; indeed, in many cases, it enables the factorial moments of $\hat{N}_{t}$ to be written down directly. Secondly, it results in expressions for these factorial moments that are both simple and probabilistically illuminating. Thirdly, it leads to asymptotic expressions for the factorial moments of $\hat{N}_{t}$ in terms of the moment matrices of the underlying Markov renewal process, which are usually readily available. By contrast, the corresponding asymptotic expressions obtained using the derived process approach require moment matrices of the derived Markov renewal process, which are usually considerably more complicated. Thus, for many counting processes, we are able to obtain simpler asymptotic results than have previously been available.

The paper is organised as follows. In Section 2, we introduce basic notation concerning Markov renewal processes and a class of associated counting process that is used in Section 3 to develop the methodology. This class comprises counting processes associated with events that are determined by the states at two successive renewal times of a Markov renewal process. It includes many counting processes previously studied in the literature as special cases. In Section 3, we present results about factorial moments and their asymptotic expansions for such counting processes. These results generalise those obtained by previous authors, and are simpler, as explained above. In Section 4, we consider specialisations to continuous-time Markov chains, for which more explicit results can be derived. In Section 5, we show that the methodology can be extended straightforwardly to deal with counting of more complicated types of event by applying it to some problems in ion channel modelling. In the final section, we offer some perspective on the present approach and indications for further work.

\section{Background and notation}

Throughout this paper vectors, matrices, and matrix-valued functions are rendered in bold; unless otherwise indicated, all vectors are column vectors and ${ }^{\top}$ denotes transpose, which is often used to express row vectors. Usually 1 denotes a column vector of ones, $\boldsymbol{I}$ an identity matrix, and $\mathbf{0}$ a matrix (or vector) of zeros, the dimensions of these being apparent from the context. Any sum is zero if vacuous. Furthermore, $1_{E}$ denotes the indicator function of a set or event $E$. Discrete- and continuous-time stochastic processes are assumed to have index sets $\mathbb{Z}_{+}=\{0,1, \ldots\}$ and $\mathbb{R}_{+}=[0, \infty)$, respectively. 


\subsection{Markov renewal processes}

Let $\left\{\left(K_{l}, R_{l}\right)\right\}=\left\{\left(K_{l}, R_{l}\right), l=0,1, \ldots\right\}$, with $R_{0}=0$, denote a time-homogeneous Markov renewal process (see, for example, Çinlar (1975a, Section 10.1) or Asmussen (2003, Section VII.4)) with state space $S \times \mathbb{R}_{+}$, where $S$ is a finite set which is taken to be $S=$ $\{1,2, \ldots, s\}$. Thus, $\left\{\left(K_{l}, R_{l}\right)\right\}$ is a bivariate Markov chain that can be specified by its semiMarkov kernel, that is by the $s \times s$ matrix function $\boldsymbol{G}(t)=\left[G_{i j}(t)\right]$, where, for each pair of states $i, j \in S$ and $l=1,2, \ldots$,

$$
G_{i j}(t)=\mathrm{P}\left(R_{l}-R_{l-1} \leq t \text { and } K_{l}=j \mid K_{l-1}=i\right), \quad t \geq 0 .
$$

Associated with any Markov renewal process $\left\{\left(K_{l}, R_{l}\right)\right\}$ are counting processes

$$
\{N(t)\}=\left\{N(t), t \in \mathbb{R}_{+}\right\} \quad \text { and } \quad\left\{N_{j}(t)\right\}=\left\{N_{j}(t), t \in \mathbb{R}_{+}\right\}, \quad j \in S,
$$

defined by $N(t)=\sup _{l \in \mathbb{Z}_{+}}\left\{l: R_{l} \leq t\right\}$ and $N_{j}(t)=\operatorname{card}\left\{l: K_{l}=j\right.$ and $\left.0<l \leq N(t)\right\}$. The process $\left\{N_{j}(t)\right\}$ counts the number of 'type- $j$ renewals' during any time interval $(0, t]$, while $\{N(t)\}$ counts the total number of renewals during any such interval. The continuous-time process $\{X(t)\}$, defined by $X(t)=K_{N(t)}$, is often termed a semi-Markov process. The process $\left\{K_{l}\right\}$ is a (homogeneous) Markov chain with transition probability matrix $\boldsymbol{P}_{K}=\left[p_{i j}^{K}\right]=\boldsymbol{G}(\infty)$. Note that if $p_{i i}^{K}=0, i \in S$, then $\left\{\left(K_{l}, R_{l}\right)\right\}$, the process $\{X(t)\}$, and the vector counting process $\left\{\left[N_{1}(t), \ldots, N_{s}(t)\right]\right\}$ are equivalent. Any Markov renewal process can be viewed as a marked (multitype) point process, with 'locations' given by $\left\{R_{l}\right\}$ and corresponding marks (types) by $\left\{K_{l}\right\}$.

Let $\boldsymbol{H}(t)=\left[H_{i j}(t)\right], t \geq 0$, denote the Markov renewal kernel of $\left\{\left(K_{l}, R_{l}\right)\right\}$, so $H_{i j}(t)=$ $\mathrm{E}\left(N_{j}(t) \mid K_{0}=i\right), t \geq 0, i, j \in S$. We assume that each $G_{i j}$ possesses a density, denoted by $g_{i j}$, and that each $H_{i j}$ has a density $h_{i j}$, with the latter having the intuitive interpretations expressed by

$$
h_{i j}(t) \mathrm{d} t=\mathrm{E}\left(N_{j}(\mathrm{~d} t) \mid K_{0}=i\right)=\mathrm{P}\left(N_{j}(\mathrm{~d} t)=1 \mid K_{0}=i\right), \quad t \geq 0, i, j \in S,
$$

where here, and in similar situations, $N_{j}(\mathrm{~d} t)$ is an abbreviation for $N_{j}(t+\mathrm{d} t)-N_{j}(t)$. For $t \geq 0$, let $\boldsymbol{g}(t)=\left[g_{i j}(t)\right]$ and $\boldsymbol{h}(t)=\left[h_{i j}(t)\right]$. Then $\boldsymbol{h}(t)=\sum_{n=1}^{\infty} \boldsymbol{g}^{(n)}(t), t \geq 0$, where $\boldsymbol{g}^{(n)}$ denotes the $n$-fold convolution of $\boldsymbol{g}$, with the convolution $\boldsymbol{f} \star \boldsymbol{g}$ of two matrix-valued functions defined as $\boldsymbol{f} \star \boldsymbol{g}(t)=\int_{0}^{t} \boldsymbol{f}(t-u) \boldsymbol{g}(u) \mathrm{d} u, t \geq 0$.

\subsection{Counting processes associated with Markov renewal processes}

Many counting processes $\left\{\hat{N}_{t}\right\}$ can be defined on a given Markov renewal process $\left\{\left(K_{l}, R_{l}\right)\right\}$. To be concrete, we assume that $\hat{N}_{t}$ denotes the number of occurrences in $(0, t]$ of some event $E$ determined by the marks at two consecutive renewal times of $\left\{\left(K_{l}, R_{l}\right)\right\}$. Thus, attention is focused on counting processes determined by events whose occurrence or nonoccurrence at a time $u=R_{l}$ is determined by the pair of states $\left(K_{l}, K_{l-1}\right)$. However, extensions render this methodology far more widely applicable, as illustrated in Section 5.

Two simple examples of an event of the above type are 'a change of state of $\{X(u)\}$ ' (i.e. $K_{l} \neq K_{l-1}$ ) and 'a jump from $A$ to $B$ ', where $A$ and $B$ are specified (not necessarily disjoint) subsets of $S$. Special cases of the latter example are 'exit from $A^{\prime}$ ' (by taking $B=A^{\prime}$, where $A^{\prime}$ denotes the set complement of $A$ ) and 'entry into $B$ ' (i.e. $A=B^{\prime}$ ).

An important special case considered by Çinlar (1975b, Section 9) is when the event is determined by the mark at a single renewal time of $\left\{\left(K_{l}, R_{l}\right)\right\}$. The simplest examples of counting processes of this type are $\{N(t)\}$ and $\left\{N_{j}(t)\right\}, j \in S$, defined above. 


\section{Factorial moments of $\left\{\hat{N}_{t}\right\}$}

\subsection{Exact results}

For $n \in \mathbb{Z}_{+}$, define $n^{[k]}=n(n-1) \cdots(n-k+1), k=1,2, \ldots$, where $n^{[0]}=1$. Now define factorial moments by $\hat{\mu}_{[k]}(t)=\mathrm{E}\left(\hat{N}_{t}^{[k]}\right), t \geq 0, k \in \mathbb{Z}_{+}$. Then we have (cf. Daley and Vere-Jones (2003, Section 5.4))

$$
\begin{aligned}
\hat{\mu}_{[k]}(t) & =\int_{0}^{t} \cdots \int_{0}^{t} \hat{m}_{[k]}\left(t_{1}, t_{2}, \ldots, t_{k}\right) \mathrm{d} t_{1} \cdots \mathrm{d} t_{k} \\
& =k ! \int_{0<t_{1}<\cdots<t_{k}<t} \cdots \int_{[k]}\left(t_{1}, t_{2}, \ldots, t_{k}\right) \mathrm{d} t_{1} \cdots d t_{k}, \quad t \geq 0, k=1,2, \ldots,
\end{aligned}
$$

where $\hat{m}_{[k]}\left(t_{1}, t_{2}, \ldots, t_{k}\right)$ is the $k$ th factorial moment density of $\left\{\hat{N}_{t}\right\}$, which has the simple intuitive interpretations

$$
\begin{gathered}
\hat{m}_{[k]}\left(t_{1}, \ldots, t_{k}\right) \mathrm{d} t_{1} \cdots \mathrm{d} t_{k}=\mathrm{E}\left(\hat{N}\left(\mathrm{~d} t_{1}\right) \cdots \hat{N}\left(\mathrm{~d} t_{k}\right)\right)=\mathrm{P}\left(\hat{N}\left(\mathrm{~d} t_{i}\right)=1, i=1, \ldots, k\right), \\
k=1,2, \ldots, t_{i} \neq t_{j}, i, j \in\{1,2, \ldots, k\}, i \neq j .
\end{gathered}
$$

The second equality in (3.1) follows using the symmetry of factorial moment densities, as can be seen from (3.2).

An event $E$ determined by the marks at two consecutive renewal times is conveniently described by a subset $T=T_{E}$ of the set $S^{2}$ of all possible (one-step) transitions, in the sense that $E$ occurs at time $u$ if, for some $l, u=R_{l}$ and $\left(K_{l-1}, K_{l}\right) \in T$. Let $A=\{j \in S:(i, j) \in T$ for some $i \in S\}$ and suppose, without loss of generality, that the states are labelled so that $A=\left\{1,2, \ldots, s_{A}\right\}$, where $s_{A}$ denotes the cardinality of $A$. Let $\hat{\boldsymbol{h}}(t)=\left[\hat{h}_{i j}(t)\right], t \geq 0$, be the $s \times s_{A}$ matrix function, where, for $i \in S$ and $j \in A, \hat{h}_{i j}(t)$ is the renewal density for the event $E$ occurring at time $t$ with $K_{l}=j$, given $K_{0}=i$. Let $B=A^{\prime}$ and partition $\hat{\boldsymbol{h}}(t)$, in the obvious fashion, as follows:

$$
\hat{\boldsymbol{h}}(t)=\left[\begin{array}{l}
\hat{\boldsymbol{h}}_{A}(t) \\
\hat{\boldsymbol{h}}_{B}(t)
\end{array}\right], \quad t \geq 0 .
$$

Then, exploiting the conditional independence along the sample paths of the underlying Markov renewal process $\left\{\left(K_{l}, R_{l}\right)\right\}$, it follows from (3.2) that

$$
\hat{m}_{[k]}\left(t_{1}, \ldots, t_{k}\right)=\boldsymbol{\alpha}^{\top} \hat{\boldsymbol{m}}_{[k]}\left(t_{1}, \ldots, t_{k}\right) \mathbf{1}, \quad k=1,2, \ldots, 0<t_{1}<\cdots<t_{k},
$$

where $\boldsymbol{\alpha}$ is the distribution of $K_{0}$ and

$$
\hat{\boldsymbol{m}}_{[k]}\left(t_{1}, \ldots, t_{k}\right)=\hat{\boldsymbol{h}}\left(t_{1}\right) \hat{\boldsymbol{h}}_{A}\left(t_{2}-t_{1}\right) \cdots \hat{\boldsymbol{h}}_{A}\left(t_{k}-t_{k-1}\right) .
$$

(This latter result is basic to our approach, yet it is simple to obtain and seemingly of independent interest as a generalisation to Markov renewal processes - in this case to derived Markov renewal processes as in Section 1 - of a result which is well known for renewal processes.) The factorial moments of $\left\{\hat{N}_{t}\right\}$ then follow by integrating as at (3.1), to yield

$$
\begin{aligned}
\hat{\mu}_{[k]}(t) & =k ! \boldsymbol{\alpha}^{\top} \int_{0}^{t}\left(\hat{\boldsymbol{h}}_{\star} \hat{\boldsymbol{h}}_{A}^{(k-1)}\right)(u) \mathrm{d} u \mathbf{1} \\
& =k ! \boldsymbol{\alpha}^{\top}\left(\hat{\boldsymbol{h}} \star \hat{\boldsymbol{h}}_{A}^{(k-1)} \star \boldsymbol{I}\right)(t) \mathbf{1}, \quad t \geq 0, k=1,2, \ldots,
\end{aligned}
$$


where $\hat{\boldsymbol{h}}^{(0)}$ is the matrix Dirac delta function concentrated on $\boldsymbol{I}_{s_{A}}$ (the $s_{A} \times s_{A}$ identity matrix), $\boldsymbol{I}=\boldsymbol{I}(u)$ denotes the matrix function which is equal to $\boldsymbol{I}_{s_{A}}$ for all $u$, and $\hat{\mu}_{[0]}(t)=1, t \geq 0$.

The so-called factorial moment generating function is

$$
\hat{\psi}_{t}(w)=\hat{\phi}_{t}(1+w)=\sum_{k=0}^{\infty} \frac{\hat{\mu}_{[k]}(t) w^{k}}{k !},
$$

where $\hat{\phi}_{t}(v)=\mathrm{E}\left(v^{\hat{N}_{t}}\right), t \geq 0, v \in[0,1]$, is the probability generating function of $\hat{N}_{t}$, and the expansion is valid in some neighbourhood of $w=0$. Furthermore,

$$
\mathrm{P}\left(\hat{N}_{t}=n\right)=\sum_{k=n}^{\infty}(-1)^{k-n}\left(\begin{array}{l}
k \\
n
\end{array}\right) \frac{\hat{\mu}_{[k]}(t)}{k !}, \quad t \geq 0, n \in \mathbb{Z}_{+} ;
$$

see, for example, Daley and Vere-Jones (2003, p. 119).

In order to use the above formulae, an expression for $\hat{\boldsymbol{h}}(t)$ is required. For $t \geq 0$, let $\boldsymbol{g}_{T}(t)=\left(\boldsymbol{g}(t) \circ \boldsymbol{J}_{T}\right) \boldsymbol{L}_{A}^{\top}$, where $\circ$ denotes a Schur (Hadamard or elementwise) product, $\boldsymbol{J}_{T}$ is the $s \times s$ matrix defined by $\boldsymbol{J}_{T}=\left[1_{T}((i, j))\right]$, and $\boldsymbol{L}_{A}$ is the $s_{A} \times s$ matrix defined, in partitioned form, by $\boldsymbol{L}_{A}=\left[\boldsymbol{I}_{s_{A}} \mathbf{0}\right]$. (Thus, the $(i, j)$ th element of $\boldsymbol{g}_{T}(t)$ is $g_{i j}(t)$ if $(i, j) \in T$ and 0 otherwise.) Then, conditioning on the last renewal of the underlying process $\left\{\left(K_{l}, R_{l}\right)\right\}$ in $[0, t)$ yields

$$
\hat{\boldsymbol{h}}(t)=\boldsymbol{g}_{T}(t)+\int_{0}^{t} \boldsymbol{h}(t-v) \boldsymbol{g}_{T}(v) \mathrm{d} v, \quad t \geq 0 .
$$

For counting processes associated with events determined by the mark at a single renewal time (so $(i, j) \in T$ if and only if $j \in A$ ), if $\boldsymbol{h}(t)$ is partitioned as

$$
\boldsymbol{h}(t)=\left[\begin{array}{ll}
\boldsymbol{h}_{A A}(t) & \boldsymbol{h}_{A B}(t) \\
\boldsymbol{h}_{B A}(t) & \boldsymbol{h}_{B B}(t)
\end{array}\right], \quad t \geq 0,
$$

then $\hat{\boldsymbol{h}}_{A}(t)=\boldsymbol{h}_{A A}(t)$ and $\hat{\boldsymbol{h}}_{B}(t)=\boldsymbol{h}_{B A}(t)$. Note the simplicity both of our results, in particular (3.3) with $\hat{h}$ given by (3.5), and their derivation when compared, for example, with those of Ball and Sansom (1987), Masuda and Sumita (1987), or Csenki (1991), all of whom considered only the special case when $\hat{N}_{t}$ counts the number of entries to $B \subset S$.

In some situations (see, for example, Section 5) it is appropriate to also specify that $X(t)=j$, and consider matrix factorial moments $\hat{\boldsymbol{M}}_{[k]}(t)=\left[\hat{M}_{i j}^{[k]}(t)\right], t \geq 0, k \in \mathbb{Z}_{+}$, where

$$
\hat{M}_{i j}^{[k]}(t)=\mathrm{E}\left(\hat{N}_{t}^{[k]} 1_{\{X(t)=j\}} \mid K_{0}=i\right), \quad t \geq 0, i \in S_{0}, j \in S, k \in \mathbb{Z}_{+} .
$$

Note that $\hat{\mu}_{[k]}(t)=\boldsymbol{\alpha}^{\top} \hat{\boldsymbol{M}}_{[k]}(t) \mathbf{1}, t \geq 0, k \in \mathbb{Z}_{+}$. Let $\boldsymbol{P}(t)=\left[P_{i j}(t)\right]$, where $P_{i j}(t)=$ $\mathrm{P}\left(X(t)=j \mid K_{0}=i\right), t \geq 0, i, j \in S$, be the transition probability function for the semi-Markov process $\{X(t)\}$, and let $\boldsymbol{P}_{A}(t)=\boldsymbol{L}_{A} \boldsymbol{P}(t)$. Then $\hat{\boldsymbol{M}}_{[0]}(t)=\boldsymbol{P}(t), t \geq 0$, and (cf. (3.3))

$$
\hat{\boldsymbol{M}}_{[k]}(t)=k !\left(\hat{\boldsymbol{h}} \star \hat{\boldsymbol{h}}_{A}^{(k-1)} \star \boldsymbol{P}_{A}\right)(t), \quad t \geq 0, k=1,2, \ldots
$$

\subsection{Asymptotic results}

For any (possibly matrix-valued) function $f$ with domain $\mathbb{R}_{+}$, denote its Laplace transform by $f^{*}(\theta)=\int_{0}^{\infty} \mathrm{e}^{-\theta t} f(t) \mathrm{d} t, \theta \geq 0$. It follows from (3.3) that

$$
\hat{\mu}_{[k]}^{*}(\theta)=k ! \theta^{-1} \boldsymbol{\alpha}^{\top} \hat{\boldsymbol{h}}^{*}(\theta)\left[\hat{\boldsymbol{h}}_{A}^{*}(\theta)\right]^{k-1} \mathbf{1}, \quad \theta>0, k=1,2, \ldots
$$


The aim is to determine the leading terms in the Laurent expansion of $\hat{\mu}_{[k]}^{*}(\theta)$, and hence obtain an asymptotic expression for $\hat{\mu}_{[k]}(t)$ as $t \rightarrow \infty$, by a Tauberian argument.

For $k=0,1, \ldots$, let $\boldsymbol{M}^{(k)}=\int_{0}^{\infty} t^{k} \mathrm{~d} \boldsymbol{G}(t)$; note that $\boldsymbol{M}^{(0)}=\boldsymbol{P}_{K}$, the transition matrix of $\left\{K_{l}\right\}$. Suppose that $\boldsymbol{M}^{(2)}$ is finite and $\left\{K_{l}\right\}$ is irreducible with equilibrium distribution $\boldsymbol{e}=\left[e_{1}, e_{2}, \ldots, e_{s}\right]^{\top}$. Let

$$
\begin{aligned}
\mu & =\boldsymbol{e}^{\top} \boldsymbol{M}^{(1)} \mathbf{1} \\
\boldsymbol{J} & =\mathbf{1} \boldsymbol{e}^{\top}, \\
\boldsymbol{Z}_{K} & =\left(\boldsymbol{I}-\boldsymbol{P}_{K}+\boldsymbol{J}\right)^{-1}=\boldsymbol{I}+\sum_{n=1}^{\infty}\left[\boldsymbol{P}_{K}^{n}-\mathbf{1 e}^{\top}\right] .
\end{aligned}
$$

Thus, $\boldsymbol{Z}_{K}$ is the fundamental matrix of $\left\{K_{l}\right\}$; see, for example, Kemeny and Snell (1976, p. 75). Now, Keilson (1969, Theorem 1) yields

$$
\boldsymbol{h}^{*}(\theta)=\theta^{-1} \mu^{-1} \boldsymbol{J}+\boldsymbol{C}+o(1) \quad \text { as } \theta \rightarrow 0+,
$$

where

$$
\boldsymbol{C}=\mu^{-1} \boldsymbol{J}\left(-\boldsymbol{M}^{(1)}+\frac{1}{2} \mu^{-1} \boldsymbol{M}^{(2)} \boldsymbol{J}\right)+\left(\boldsymbol{Z}_{K}-\mu^{-1} \boldsymbol{J} \boldsymbol{M}^{(1)} \boldsymbol{Z}_{K}\right)\left(\boldsymbol{M}^{(0)}-\mu^{-1} \boldsymbol{M}^{(1)} \boldsymbol{J}\right) .
$$

(See Hunter (1969, Theorem 3.3) for an alternative but equivalent expression for $\boldsymbol{C}$.)

Taking Laplace transforms of both sides of (3.5) yields

$$
\hat{\boldsymbol{h}}^{*}(\theta)=\left[\boldsymbol{I}+\boldsymbol{h}^{*}(\theta)\right] \boldsymbol{g}_{T}^{*}(\theta), \quad \theta>0 .
$$

For $k=0,1, \ldots$, let $\boldsymbol{M}_{T}^{(k)}=\left(\boldsymbol{M}^{(k)} \circ \boldsymbol{J}_{T}\right) \boldsymbol{L}_{A}^{\top}$. Then

$$
\boldsymbol{g}_{T}^{*}(\theta)=\boldsymbol{M}_{T}^{(0)}-\boldsymbol{M}_{T}^{(1)} \theta+o(\theta) \quad \text { as } \theta \rightarrow 0+.
$$

Substituting (3.8) and (3.10) into (3.9) yields

$$
\hat{\boldsymbol{h}}^{*}(\theta)=\theta^{-1} \mu^{-1} \boldsymbol{U}_{T}+\boldsymbol{V}_{T}+o(1) \quad \text { as } \theta \rightarrow 0+,
$$

where $\boldsymbol{U}_{T}=\boldsymbol{J} \boldsymbol{M}_{T}^{(0)}$ and $\boldsymbol{V}_{T}=(\boldsymbol{I}+\boldsymbol{C}) \boldsymbol{M}_{T}^{(0)}-\mu^{-1} \boldsymbol{J} \boldsymbol{M}_{T}^{(1)}$. Then we obtain

$$
\hat{\boldsymbol{h}}_{A}^{*}(\theta)=\theta^{-1} \mu^{-1} \boldsymbol{U}_{T A}+\boldsymbol{V}_{T A}+o(1) \quad \text { as } \theta \rightarrow 0+,
$$

where $\boldsymbol{U}_{T A}=\boldsymbol{L}_{A} \boldsymbol{U}_{T}$ and $\boldsymbol{V}_{T A}=\boldsymbol{L}_{A} \boldsymbol{V}_{T}$.

Let $e_{T}=\boldsymbol{e}^{\top} \boldsymbol{M}_{T}^{(0)} \mathbf{1}$ be the equilibrium probability that a point of the underlying Markov renewal process corresponds to an occurrence of the event $E$. Noting that $\boldsymbol{U}_{T A}^{k}=e_{T}^{k-1} \boldsymbol{U}_{T A}$, $k=1,2 \ldots$, it follows from (3.12) that, for $k=2,3, \ldots$,

$$
\left\{\hat{\boldsymbol{h}}_{A}^{*}(\theta)\right\}^{k}=(\theta \mu)^{-k} e_{T}^{k-1} \boldsymbol{U}_{T A}+(\theta \mu)^{-(k-1)} e_{T}^{k-2} \boldsymbol{B}_{k-2}^{T}+o\left(\theta^{-(k-1)}\right) \quad \text { as } \theta \rightarrow 0+,
$$

where $\boldsymbol{B}_{k}^{T}=\boldsymbol{U}_{T A} \boldsymbol{V}_{T A}+\boldsymbol{V}_{T A} \boldsymbol{U}_{T A}+k e_{T}^{-1} \boldsymbol{U}_{T A} \boldsymbol{V}_{T A} \boldsymbol{U}_{T A}$. Observe that

$$
\begin{aligned}
\boldsymbol{\alpha}^{\top} \boldsymbol{U}_{T} \mathbf{1} & =e_{T}, & \boldsymbol{\alpha}^{\top} \boldsymbol{U}_{T} \boldsymbol{U}_{T A} \mathbf{1} & =e_{T}^{2}, \\
\boldsymbol{\alpha}^{\top} \boldsymbol{U}_{T} \boldsymbol{V}_{T A} \mathbf{1} & =\boldsymbol{e}^{\top} \boldsymbol{M}_{T}^{(0)} \boldsymbol{V}_{T A} \mathbf{1}, & \boldsymbol{\alpha}^{\top} \boldsymbol{V}_{T} \boldsymbol{U}_{T A} \mathbf{1} & =e_{T} \boldsymbol{\alpha}^{\top} \boldsymbol{V}_{T} \mathbf{1}, \\
\boldsymbol{\alpha}^{\top} \boldsymbol{U}_{T} \boldsymbol{B}_{k}^{T} \mathbf{1} & =(k+2) e_{T} \boldsymbol{e}^{\top} \boldsymbol{M}_{T}^{(0)} \boldsymbol{V}_{T A} \mathbf{1}, & k & =0,1, \ldots
\end{aligned}
$$


Treating the cases $k=1, k=2$, and $k \geq 3$ separately, substituting (3.11)-(3.13) into (3.7), and using the above relations yields, after a little algebra,

$$
\hat{\mu}_{[k]}^{*}(\theta)=k ! \theta^{-(k+1)} \mu^{-k} e_{T}^{k}+k ! \theta^{-k} \mu^{-(k-1)} e_{T}^{k-1} b_{k}^{T}+o\left(\theta^{-(k-1)}\right) \quad \text { as } \theta \rightarrow 0+,
$$

for $k=1,2, \ldots$, where $b_{k}^{T}=\boldsymbol{\alpha}^{\top} \boldsymbol{V}_{T} \mathbf{1}+(k-1) e_{T}^{-1} \boldsymbol{e}^{\top} \boldsymbol{M}_{T}^{(0)} \boldsymbol{V}_{T A} \mathbf{1}, k=1,2, \ldots$ Hence, by a standard Tauberian result, for $k=1,2, \ldots$,

$$
\hat{\mu}_{[k]}(t)=\mu^{-k} e_{T}^{k} t^{k}+k \mu^{-(k-1)} e_{T}^{k-1} b_{k}^{T} t^{k-1}+o\left(t^{-(k-1)}\right) \quad \text { as } t \rightarrow \infty .
$$

This expression both simplifies and extends previous results; for example, see Masuda and Sumita (1987, Theorem 4.3), which gave similar results for the number of entries to $B \subset S$ in terms of moment matrices of a derived process.

Suppose that information concerning the state $X(t)$ is also required. Taking the Laplace transform of (3.6) yields

$$
\hat{\boldsymbol{M}}_{[k]}^{*}(\theta)=k ! \hat{\boldsymbol{h}}^{*}(\theta)\left[\hat{\boldsymbol{h}}_{A}^{*}(\theta)\right]^{k-1} \boldsymbol{P}_{A}^{*}(\theta), \quad \theta>0, k=1,2, \ldots
$$

Also, $\hat{\boldsymbol{M}}_{[0]}^{*}(\theta)=\boldsymbol{P}^{*}(\theta), \theta>0$. An asymptotic expression for $\hat{\boldsymbol{M}}_{[k]}(t)$ can be derived from (3.14), using a similar argument to the above and the expansion which is now derived for $\boldsymbol{P}^{*}(\theta)$. Let $\boldsymbol{F}(t)$ be the $s \times s$ diagonal matrix with $F_{i i}(t)=\mathrm{P}\left(R_{1}>t \mid K_{0}=i\right), i \in S$. Then $\boldsymbol{F}(t)=\boldsymbol{I}-\operatorname{diag}\left\{\int_{0}^{t} \boldsymbol{g}(u) \mathbf{1} \mathrm{d} u\right\}$ where, for a vector $\boldsymbol{v}, \operatorname{diag}\{\boldsymbol{v}\}$ denotes the diagonal matrix whose entries on the diagonal are those of $\boldsymbol{v}$. Conditioning on the time of the last renewal in $[0, t)$, we obtain

$$
\boldsymbol{P}(t)=\boldsymbol{F}(t)+\int_{0}^{t} \boldsymbol{h}(u) \boldsymbol{F}(t-u) \mathrm{d} u, \quad t \geq 0,
$$

so

$$
\boldsymbol{P}^{*}(\theta)=\left[\boldsymbol{I}+\boldsymbol{h}^{*}(\theta)\right] \boldsymbol{F}^{*}(\theta), \quad \theta>0 .
$$

Now,

$$
\boldsymbol{F}^{*}(\theta)=\theta^{-1} \boldsymbol{I}-\operatorname{diag}\left\{\theta^{-1} \boldsymbol{g}^{*}(\theta) \mathbf{1}\right\}=\operatorname{diag}\left\{\boldsymbol{M}^{(1)} \mathbf{1}\right\}+o(1) \quad \text { as } \theta \rightarrow 0+.
$$

Substituting (3.8) and (3.16) into (3.15) yields, after a little algebra,

$$
\boldsymbol{P}^{*}(\theta)=\theta^{-1} \mu^{-1} \boldsymbol{J} \operatorname{diag}\left\{\boldsymbol{M}^{(1)} \mathbf{1}\right\}+(\boldsymbol{I}+\boldsymbol{C}) \operatorname{diag}\left\{\boldsymbol{M}^{(1)} \mathbf{1}\right\}+o(1) \quad \text { as } \theta \rightarrow 0+.
$$

\section{Specialisation to continuous-time Markov chains}

Suppose now that $\{X(t)\}$ is a homogeneous continuous-time Markov chain with finite state space $S=\{1,2, \ldots, s\}$ and $s \times s$ transition rate matrix $\boldsymbol{Q}=\left[q_{i j}\right]$. The associated Markov renewal process $\left\{\left(K_{l}, R_{l}\right)\right\}$, which we assume for convenience to have $R_{l} \neq R_{l+1}$ for each $l$, has Markov renewal kernel density $\boldsymbol{g}(t)$, given by $g_{i j}(t)=q_{i j} \mathrm{e}^{-q_{i} t}$ if $i \neq j$ and 0 otherwise, where $q_{i}=-q_{i i}=\sum_{j \neq i} q_{i j}, i \in S$.

An event $E$ of the type introduced in Section 2.2 is now determined by knowledge of $X(u)$ and $X(u-)$, where $u$ is a jump time of $\{X(t)\}$. As previously, $\hat{N}_{t}$ denotes the number of occurrences of the event $E$ during the time interval $(0, t]$, and $T$ the set of (one-step) transitions which can be used to describe $E$.

The Markov property implies that, in the notation of Section $3.1, \hat{\boldsymbol{h}}(t)=\mathrm{e}^{\boldsymbol{Q} t} \boldsymbol{Q}_{T} \boldsymbol{L}_{A}^{\top}$, $\hat{\boldsymbol{h}}_{A}(t)=\boldsymbol{L}_{A} \mathrm{e}^{\boldsymbol{Q} t} \boldsymbol{Q}_{T} \boldsymbol{L}_{A}^{\top}$, and $\boldsymbol{P}(t)=\mathrm{e}^{\boldsymbol{Q} t}$, where $\mathrm{e}^{\boldsymbol{Q} t}=\sum_{j=0}^{\infty} t^{j} \boldsymbol{Q}^{j} / j !, t \geq 0$, is the usual 
matrix exponential (see, for example, Bellman (1970, p. 169)) and $\boldsymbol{Q}_{T}=\boldsymbol{Q} \circ \boldsymbol{J}_{T}$. Note that $\boldsymbol{Q}_{T} \boldsymbol{L}_{A}^{\top} \boldsymbol{L}_{A}=\boldsymbol{Q}_{T}$. It then follows from (3.6) that

$$
\hat{\boldsymbol{M}}_{[k]}(t)=k !\left(\tilde{\boldsymbol{h}}^{(k)} \star \boldsymbol{P}\right)(t), \quad t \geq 0, k=0,1, \ldots,
$$

where $\tilde{\boldsymbol{h}}(t)=\mathrm{e}^{\boldsymbol{Q} t} \boldsymbol{Q}_{T}$. (Note that (4.1) could have been written down directly using matrix versions of (3.1) and (3.2), and the Markov property of $\{X(t)\}$.)

Taking Laplace transforms of both sides of (4.1) yields

$$
\hat{\boldsymbol{M}}_{[k]}^{*}(\theta)=k !\left[\tilde{\boldsymbol{h}}^{*}(\theta)\right]^{k} \boldsymbol{P}^{*}(\theta), \quad \theta>0, k=0,1, \ldots,
$$

where $\tilde{\boldsymbol{h}}^{*}(\theta)=\boldsymbol{P}^{*}(\theta) \boldsymbol{Q}_{T}$ and $\boldsymbol{P}^{*}(\theta)=(\theta \boldsymbol{I}-\boldsymbol{Q})^{-1}$. Let $\hat{\boldsymbol{\Psi}}_{t}(w)=\sum_{k=0}^{\infty} \hat{\boldsymbol{M}}_{[k]}(t) w^{k} / k$ !, $t>0$, be the matrix factorial moment generating function of $\hat{N}_{t}$. Then, from (4.2), the Laplace transform of $\hat{\boldsymbol{\Psi}}_{t}(w)$ is given by $\hat{\boldsymbol{\Psi}}^{*}(\theta, w)=\left(\theta \boldsymbol{I}-\boldsymbol{Q}-w \boldsymbol{Q}_{T}\right)^{-1}, \theta>0$, for $w$ in some neighbourhood of $w=0$. Inversion of this Laplace transform yields $\hat{\mathbf{\Psi}}_{t}(w)=\exp ([\boldsymbol{Q}+$ $\left.w \boldsymbol{Q}_{T}\right] t$ ), so the matrix probability generating function of $\hat{N}_{t}$ is

$$
\hat{\boldsymbol{\Phi}}_{t}(v)=\exp \left(\left[\boldsymbol{Q}+(v-1) \boldsymbol{Q}_{T}\right] t\right), \quad t \geq 0,
$$

at least for $v \in[0,1]$; cf. Darroch and Morris (1967) and Neuts (1995, p. 284), who, in effect, derived (4.3) using a forward equation. In principle, a compact expression can be given for $\hat{\boldsymbol{M}}_{[k]}(t)$ in terms of the $k$ th derivative of this generating function. In practice, however, since $\boldsymbol{Q}$ and $\boldsymbol{Q}_{T}$ do not in general commute (and so the matrix exponential cannot be factorised), such an expression does not seem useful.

Returning to the factorial moments, setting $k=1$ in (4.1) yields

$$
\hat{\boldsymbol{M}}_{[1]}(t)=\int_{0}^{t} \mathrm{e}^{\boldsymbol{Q} u} \boldsymbol{Q}_{T} \mathrm{e}^{\boldsymbol{Q}(t-u)} \mathrm{d} u, \quad t \geq 0,
$$

though, in general, there appears to be no simple expression even for this integral. More explicit results are available when information about $X(t)$ is not required or when the initial distribution $\boldsymbol{\alpha}$ is chosen suitably, as we now explore when $\{X(t)\}$ is irreducible.

\subsection{Irreducible chains}

For $t \geq 0$ and $k=0,1, \ldots$, let $\hat{\boldsymbol{\mu}}_{[k]}(t)=\hat{\boldsymbol{M}}_{[k]}(t) \mathbf{1}, \hat{\boldsymbol{\mu}}_{[k]}(t, \boldsymbol{\alpha})=\boldsymbol{\alpha}^{\top} \hat{\boldsymbol{M}}_{[k]}(t)$, and $\hat{\mu}_{[k]}(t, \boldsymbol{\alpha})=\boldsymbol{\alpha}^{\top} \hat{\boldsymbol{M}}_{[k]}(t) \mathbf{1}$. Thus, for example, the $i$ th component of $\hat{\boldsymbol{\mu}}_{[k]}(t)$ is the $k$ th factorial moment of $\hat{N}_{t}$, given that $X(0)=i$, and $\hat{\mu}_{[k]}(t, \alpha)$ is the $k$ th factorial moment of $\hat{N}_{t}$ when the distribution of $X(0)$ is given by $\boldsymbol{\alpha}$.

4.1.1. Exact results. Suppose that $\{X(t)\}$ is irreducible, with equilibrium distribution $\pi=$ $\left[\pi_{1}, \pi_{2}, \ldots, \pi_{s}\right]^{\top}$. Let $\boldsymbol{Z}=\int_{0}^{\infty}\left(\mathrm{e}^{\boldsymbol{Q} t}-\mathbf{1} \boldsymbol{\pi}^{\top}\right) \mathrm{d} t=\left(\mathbf{1} \boldsymbol{\pi}^{\top}-\boldsymbol{Q}\right)^{-1}-\mathbf{1} \boldsymbol{\pi}^{\top}$ denote the fundamental matrix of $\{X(t)\}$ (see, for example, Keilson (1979, p. 107)). Then

$$
\int_{0}^{t} \mathrm{e}^{\boldsymbol{Q} u} \mathrm{~d} u=\boldsymbol{Z}\left(\boldsymbol{I}-\mathrm{e}^{\boldsymbol{Q} t}\right)+\mathbf{1} \boldsymbol{\pi}^{\top} t, \quad t \geq 0 ;
$$

see Ball et al. (1994, Theorem 3.4) and Neuts (1995, p. 245).

Using $\boldsymbol{P}(t) \mathbf{1}=\mathrm{e}^{\boldsymbol{Q}^{t}} \mathbf{1}=\mathbf{1}$, it then follows from (4.4) that, for $t \geq 0$,

$$
\hat{\boldsymbol{\mu}}_{[1]}(t)=\int_{0}^{t} \mathrm{e}^{\boldsymbol{Q} u} \boldsymbol{Q}_{T} \mathbf{1} \mathrm{d} u=\boldsymbol{Z}\left(\boldsymbol{I}-\mathrm{e}^{\boldsymbol{Q} t}\right) \boldsymbol{Q}_{T} \mathbf{1}+\mathbf{1} \boldsymbol{\pi}^{\top} \boldsymbol{Q}_{T} \mathbf{1} t
$$


and, setting $k=2$ in (4.1), it follows that

$$
\hat{\boldsymbol{\mu}}_{[2]}(t)=2 \int_{0}^{t} \mathrm{e}^{\boldsymbol{Q ( t - u )}} \boldsymbol{Q}_{T}\left[\boldsymbol{Z}\left(\boldsymbol{I}-\mathrm{e}^{\boldsymbol{Q} u}\right) \boldsymbol{Q}_{T} \mathbf{1}+\mathbf{1} \boldsymbol{\pi}^{\top} \boldsymbol{Q}_{T} \mathbf{1} u\right] \mathrm{d} u .
$$

The right-hand side of (4.7) involves an integral of the form

$$
\int_{0}^{t} \mathrm{e}^{-\boldsymbol{Q} u} \boldsymbol{Q}_{T} \boldsymbol{Z} \mathrm{e}^{\boldsymbol{Q} u} \mathrm{~d} u
$$

for which no simple expression exists. The remaining integrals in (4.7) are straightfoward to evaluate using (4.5).

Suppose now that $\{X(t)\}$ is in equilibrium. Then it follows from (4.1) that $\hat{\boldsymbol{\mu}}_{[k]}(t, \pi)=$ $k ! \boldsymbol{\pi}^{\top}\left(\tilde{\boldsymbol{h}}^{(k)} \star \boldsymbol{P}\right)(t), t \geq 0, k=0,1, \ldots$ Thus,

$$
\begin{aligned}
\hat{\boldsymbol{\mu}}_{[1]}(t, \boldsymbol{\pi}) & =\int_{0}^{t} \boldsymbol{\pi}^{\top} \mathrm{e}^{\boldsymbol{Q}(t-u)} \boldsymbol{Q}_{T} \mathrm{e}^{\boldsymbol{Q} u} \mathrm{~d} u \\
& =\boldsymbol{\pi}^{\top} \boldsymbol{Q}_{T} \int_{0}^{t} \mathrm{e}^{\boldsymbol{Q} u} \mathrm{~d} u \\
& =\boldsymbol{\pi}^{\top} \boldsymbol{Q}_{T}\left[\boldsymbol{Z}\left(\boldsymbol{I}-\mathrm{e}^{\boldsymbol{Q} t}\right)+\mathbf{1} \boldsymbol{\pi}^{\top} t\right], \quad t \geq 0 .
\end{aligned}
$$

Again, $\hat{\boldsymbol{\mu}}_{[2]}(t, \pi)$ will involve an integral of the form (4.8). Furthermore, since $\hat{\mu}_{[k]}(t, \pi)=$ $\boldsymbol{\pi}^{\top} \hat{\boldsymbol{\mu}}_{[k]}(t), t \geq 0, k=1,2, \ldots$, (4.6) implies that $\hat{\mu}_{[1]}(t, \boldsymbol{\pi})=\boldsymbol{\pi}^{\top} \boldsymbol{Q}_{T} \mathbf{1} t, t \geq 0$, and (4.7) implies that

$$
\begin{aligned}
\hat{\mu}_{[2]}(t, \boldsymbol{\pi}) & =2 \int_{0}^{t} \boldsymbol{\pi}^{\top} \mathrm{e}^{\boldsymbol{Q ( t - u )}} \boldsymbol{Q}_{T}\left[\boldsymbol{Z}\left(\boldsymbol{I}-\mathrm{e}^{\boldsymbol{Q} u}\right) \boldsymbol{Q}_{T} \mathbf{1}+\mathbf{1} \boldsymbol{\pi}^{\top} \boldsymbol{Q}_{T} \mathbf{1} u\right] \mathrm{d} u \\
& =2 \boldsymbol{\pi}^{\top} \boldsymbol{Q}_{T} \boldsymbol{Z} \boldsymbol{Q}_{T} \mathbf{1} t-2 \boldsymbol{\pi}^{\top} \boldsymbol{Q}_{T} \boldsymbol{Z}\left[\boldsymbol{Z}\left(\boldsymbol{I}-\mathrm{e}^{\boldsymbol{Q} t}\right)+\mathbf{1} \boldsymbol{\pi}^{\top}\right] \boldsymbol{Q}_{T} \mathbf{1}+\left(\boldsymbol{\pi}^{\top} \boldsymbol{Q}_{T} \mathbf{1}\right)^{2} t^{2} \\
& =2 \boldsymbol{\pi}^{\top} \boldsymbol{Q}_{T} \boldsymbol{Z} \boldsymbol{Q}_{T} \mathbf{1} t-2 \boldsymbol{\pi}^{\top} \boldsymbol{Q}_{T} \boldsymbol{Z}^{2}\left(\boldsymbol{I}-\mathrm{e}^{\boldsymbol{Q}^{t}}\right) \boldsymbol{Q}_{T} \mathbf{1}+\left(\boldsymbol{\pi}^{\top} \boldsymbol{Q}_{T} \mathbf{1}\right)^{2} t^{2}, \quad t \geq 0,
\end{aligned}
$$

as $\boldsymbol{Z 1}=\mathbf{0}$. Note that $\mu_{[3]}(t, \boldsymbol{\mu})$ involves an integral similar to (4.8).

When $Q$ admits a spectral representation, the recursive algorithm of Hawkes et al. (1990, Equation (3.18)) is easily modified to provide a means of computing $\left(\tilde{\boldsymbol{h}}^{(k)} \star \boldsymbol{P}\right)(t)$ and, hence, $\hat{\boldsymbol{M}}_{[k]}(t), t \geq 0, k \in \mathbb{Z}_{+}$.

4.1.2. Asymptotic results. As in Section 3.2, the aim is to derive the leading terms in the Laurent expansion of $\hat{\boldsymbol{M}}_{[k]}^{*}(\theta)$ and hence obtain an asymptotic expression for $\hat{\boldsymbol{M}}_{[k]}(t)$ as $t \rightarrow \infty$. Recall that $\boldsymbol{P}(t)=\mathrm{e}^{\boldsymbol{Q} t}$. Taking the Laplace transform of (4.5) yields

$$
\theta^{-1} \boldsymbol{P}^{*}(\theta)=\theta^{-1} \boldsymbol{Z}-\boldsymbol{Z} \boldsymbol{P}^{*}(\theta)+\theta^{-2} \mathbf{1} \boldsymbol{\pi}^{\top}, \quad \theta \geq 0 .
$$

Thus, $(\boldsymbol{I}+\theta \boldsymbol{Z}) \boldsymbol{P}^{*}(\theta)=\boldsymbol{Z}+\theta^{-1} \mathbf{1} \boldsymbol{\pi}^{\top}$ so, after some algebra and the use of $\boldsymbol{Z 1}=\mathbf{0}$, it follows that $\boldsymbol{P}^{*}(\theta)=\theta^{-1} \mathbf{1} \boldsymbol{\pi}^{\top}+\boldsymbol{Z}+o(1)$ as $\theta \rightarrow 0+$. Hence, from (4.2), for $k=1,2, \ldots$,

$$
\begin{aligned}
\hat{\boldsymbol{M}}_{[k]}^{*}(\theta) & =k !\left(\boldsymbol{P}^{*}(\theta) \boldsymbol{Q}_{T}\right)^{k} \boldsymbol{P}^{*}(\theta) \\
& =k !\left[\left(\theta^{-1} \mathbf{1} \boldsymbol{\pi}^{\top}+\boldsymbol{Z}+o(1)\right) \boldsymbol{Q}_{T}\right]^{k}\left(\theta^{-1} \mathbf{1} \boldsymbol{\pi}^{\top}+\boldsymbol{Z}+o(1)\right) \\
& =k ! \theta^{-(k+1)}\left(\mathbf{1} \boldsymbol{\pi}^{\top} \boldsymbol{Q}_{T}\right)^{k} \mathbf{1} \boldsymbol{\pi}^{\top}+k ! \theta^{-k} \boldsymbol{S}_{k}+o\left(\theta^{-k}\right) \quad \text { as } \theta \rightarrow 0+,
\end{aligned}
$$

where $\boldsymbol{S}_{k}=\left(\mathbf{1} \boldsymbol{\pi}^{\top} \boldsymbol{Q}_{T}\right)^{k} \boldsymbol{Z}+\sum_{l=0}^{k-1}\left(\mathbf{1} \boldsymbol{\pi}^{\top} \boldsymbol{Q}_{T}\right)^{l} \boldsymbol{Z} \boldsymbol{Q}_{T}\left(\mathbf{1} \boldsymbol{\pi}^{\top} \boldsymbol{Q}_{T}\right)^{k-1-l} \mathbf{1} \boldsymbol{\pi}^{\top}$. Hence,

$$
\hat{\boldsymbol{M}}_{[k]}(t)=\left(\mathbf{1} \boldsymbol{\pi}^{\top} \boldsymbol{Q}_{T}\right)^{k} \mathbf{1} \boldsymbol{\pi}^{\top} t^{k}+k \boldsymbol{S}_{k} t^{k-1}+o\left(t^{k-1}\right) \quad \text { as } t \rightarrow \infty, \quad k=1,2, \ldots,
$$

and asymptotic results follow immediately for the other forms of factorial moments. 


\section{Applications to ion channel modelling}

This section illustrates that the methodology developed in Sections 3 and 4 generalises easily to counting processes associated with more complex events, by showing that it provides simple, novel solutions to some problems in ion channel modelling.

\subsection{Background}

The gating mechanism of a single ion channel is usually modelled by a continuous-time Markov chain $\{X(t)\}$ with finite state space $S=\{1,2, \ldots, s\}$ (see, for example, Colquhoun and Hawkes (1982)). The state space is partitioned into two classes: the open class $O=$ $\left\{1,2, \ldots, s_{O}\right\}$, which corresponds to the receptor channel being open, and the closed class $C=\left\{s_{O}+1, s_{O}+2, \ldots, s\right\}$, which corresponds to the receptor channel being closed. Thus, $s_{O}$ is the number of states in the open class. At any given time it is, in principle, possible to observe which class $\{X(t)\}$ is in, but not which state within that class. In practice, the sequence of open and closed sojourns of the channel is often 'reconstructed' from the recorded current flowing across the channel by using a filter and an associated threshold crossing algorithm, which results in the loss of very short sojourns in either the open or closed classes of state. This phenomenon is known as time interval omission and is usually modelled by assuming that any open or closed sojourn of duration less than some critical value $\tau$ fails to be detected; see, for example, Hawkes et al. (1990), who determined an exact expression for the probability density function of the length of such an observed open sojourn using term-by-term inversion of an infinite series for its Laplace transform.

Recordings of single channels often show periods of repetitive open activity, known as bursts, which are noticeably separated from other such periods. Specifically, a burst is a sequence of times during which the channel is open together with the intervening short closedtimes (called gaps), with neighbouring bursts separated by much longer closed-times (called interburst sojourns). A burst may be defined in two different ways: theoretical bursts, in which the closed states are partitioned into short-lived and long-lived states, and empirical bursts, in which closed-times are classified as long or short according to whether or not they exceed some specified critical time $t_{\mathrm{c}}$ (see, for example, Colquhoun and Hawkes (1982)). Properties of the number of theoretical and empirical bursts commencing in the time interval $(0, t]$ were investigated by Ball and Davies (1997) and Ball (1997), respectively.

\subsection{Counting empirical bursts}

Suppose that the transition rate matrix $Q$ of $\{X(t)\}$ is partitioned as follows:

$$
\boldsymbol{Q}=\left[\begin{array}{ll}
\boldsymbol{Q}_{O O} & \boldsymbol{Q}_{O C} \\
\boldsymbol{Q}_{C O} & \boldsymbol{Q}_{C C}
\end{array}\right] .
$$

Observe that an empirical burst commences each time $\{X(t)\}$ makes a transition to $O$ that is immediately preceded by a sojourn in $C$ of duration exceeding $t_{\mathrm{c}}$. Thus, since $\{X(t)\}$ is Markov, the Markov renewal density of such an event is

$$
\tilde{\boldsymbol{h}}(t)=\mathrm{e}^{\boldsymbol{Q}\left(t-t_{\mathrm{c}}\right)} \boldsymbol{Q}_{T}, \quad t \geq \tau,
$$

where

$$
\boldsymbol{Q}_{T}=\left[\begin{array}{cc}
\mathbf{0} & \mathbf{0} \\
\mathrm{e}^{\boldsymbol{Q}_{C C t_{\mathrm{c}}}} \boldsymbol{Q}_{C O} & \mathbf{0}
\end{array}\right],
$$

and $\hat{\boldsymbol{M}}_{[k]}(t)$ (the matrix $k$ th factorial moment of $\hat{N}_{t}$, the number of empirical bursts commencing in $(0, t])$ is given by (4.1), with this choice of $\tilde{\boldsymbol{h}}$, and $\boldsymbol{P}(t)=\mathrm{e}^{\boldsymbol{Q} t}$ as before. 
For $t \geq 0$ and $n=0,1, \ldots$, let $\boldsymbol{p}^{(n)}(t)=\left[p_{i j}^{(n)}(t)\right]$, where

$$
p_{i j}^{(n)}(t)=\mathrm{P}\left(\hat{N}_{t}=n \text { and } X(t)=j \mid X(0)=i\right), \quad t \geq 0, i, j \in S .
$$

Thus, $\boldsymbol{p}^{(n)}(t)$ is the matrix probability mass function of $\hat{N}_{t}$. Note that $\boldsymbol{p}^{(k)}(t)=\mathbf{0}$ and $\hat{\boldsymbol{M}}_{[k]}(t)=$ $\mathbf{0}$ for $t \in\left[0, k t_{\mathrm{c}}\right)$, since $t$ must be at least $k t_{\mathrm{c}}$ for $k$ empirical bursts to start in $(0, t]$. Hence, with $\lfloor\cdot\rfloor$ denoting the integer-part function, the matrix analogue of (3.4) gives

$$
\begin{aligned}
\boldsymbol{p}^{(n)}(t) & =\sum_{k=n}^{\infty}(-1)^{k-n}\left(\begin{array}{l}
k \\
n
\end{array}\right) \frac{\hat{\boldsymbol{M}}_{[k]}(t)}{k !} \\
& =\sum_{k=n}^{\lfloor t / \tau\rfloor}(-1)^{k-n}\left(\begin{array}{l}
k \\
n
\end{array}\right)\left(\tilde{\boldsymbol{h}}^{(k)} \star \boldsymbol{P}\right)(t), \quad t \geq 0, n \in \mathbb{Z}_{+} .
\end{aligned}
$$

Equation (5.2) was obtained previously by Ball (1997, Corollary 4.4), using a lengthy argument involving solution of a backward delay differential equation for the corresponding probability generating function.

There is a simple connection between empirical bursts and observed open sojourns, in that within an observed open sojourn there can be no closed sojourn of duration exceeding $\tau$; see Ball (1997, Section 5). Let $\tilde{\boldsymbol{p}}^{(n)}(t)$ be given by (5.2), with $t_{\mathrm{c}}$ replaced by $\tau$, and partition $\tilde{\boldsymbol{p}}^{(n)}(t)$ in a similar manner to $Q$ in (5.1). Then, the Markov property of $\{X(t)\}$ implies that the (matrix) probability density function of the length of an observed open sojourn is given by

$$
\boldsymbol{f}_{O C}(t)=\boldsymbol{D}_{O}^{-1} \mathrm{e}^{\boldsymbol{Q}_{O O} \tau} \tilde{\boldsymbol{p}}_{O O}^{(0)}(t-\tau) \boldsymbol{Q}_{O C} \boldsymbol{D}_{C}, \quad t \geq \tau,
$$

where $\boldsymbol{D}_{O}=\operatorname{diag}\left\{\mathrm{e}^{\boldsymbol{Q}_{O O} \tau} \mathbf{1}\right\}$ and $\boldsymbol{D}_{C}=\operatorname{diag}\left\{\boldsymbol{e}_{C C} \boldsymbol{1}\right\}$; cf. Ball (1997, Equation (5.1)). (Note that, for example, the elements of $\mathrm{e}^{\boldsymbol{Q}_{O o} \tau} \mathbf{1}$ give the probability that an (actual) open sojourn is of duration at least $\tau$ for the various initial states.) The expression for $f_{O C}(t)$ obtained by Hawkes et al. (1990), referred to in the first paragraph of Section 5.1, follows by setting $n=0$ in (5.2) and substituting this into (5.3).

The above arguments easily extend to the case in which $\{X(t)\}$ is semi-Markov, though when deriving an expression for $\boldsymbol{f}_{O C}(t)$, as $\{X(t)\}$ is not Markov, $\tilde{\boldsymbol{p}}^{(n)}(t)$ needs to be replaced by an associated density corresponding to $\{X(t)\}$ making a transition at time $t$; cf. Ball et al. (1993).

\subsection{Counting theoretical bursts}

Let $C_{S}$ and $C_{L}$ denote the classes of short-lived and long-lived closed states, respectively, so $C=C_{S} \cup C_{L}$. Counting the number of theoretical bursts commencing in $(0, t]$ is equivalent to counting the number of transitions $\{X(t)\}$ makes to $O$ either directly from $C_{L}$ or from $C_{S}$, having been in $C_{L}$ immediately before moving to $C_{S}$. Following Ball and Davies (1997), augment the state space by replacing each state in $C_{S}$ by two states, labelled according to whether the previous class was $O$ or $C_{L}$. Group these new states together in new classes denoted, in an obvious manner, by $C_{S O}$ and $C_{S L}$. For the aggregated process on this enlarged state space, having classes $O, C_{S O}, C_{S L}$, and $C_{L}$, to count theoretical bursts requires only that we count transitions from $C_{S L} \cup C_{L}$ to $O$. Thus, the problem is reduced to a counting problem of a type already dealt with in Section 4. Note that the extension to the case when $\{X(t)\}$ is semi-Markov is immediate, using results from Section 3. 


\section{Concluding remarks}

In this paper, we have developed a simple approach to properties of counts $\hat{N}_{t}$ of the number of occurrences in $(0, t]$ of some event defined on an underlying Markov renewal process. The approach provides a unification of previous approaches to such counting problems, and a more direct route to the (factorial) moments of the counts and their asymptotic properties. By first determining the factorial moments and then the corresponding probability generating function, the usual route taken to such counting problems is reversed. Furthermore, our route is simpler and more efficient, especially as regards the determination of factorial moments, which in many investigations have been the quantities of primary interest and often can be written down directly using the present methodology. Moreover, our results are often easier to use in practice, as they are expressed in terms of the underlying, rather than a derived, Markov renewal process.

No details have been given of possible derivations from the probability generating function of other properties, such as those of waiting times to the $k$ th occurrence of the event under consideration,. Some such details were considered by Darroch and Morris (1967) in cases where the underlying process is a discrete- or continuous-time Markov chain. The methodology described in Section 3 can be generalised to cover Markov renewal processes in discrete time (and thereby discrete-time Markov chains), and also terminating Markov renewal processes. In the latter case, $\hat{N}_{t}$ often has a matrix-geometric distribution as $t \rightarrow \infty$, and results similar to those of Section 4.1.1 are available using $\int_{0}^{t} \mathrm{e}^{\boldsymbol{Q} u} \mathrm{~d} u=\boldsymbol{Q}^{-1}\left(\mathrm{e}^{\boldsymbol{Q} t}-\boldsymbol{I}\right)$, since $\boldsymbol{Q}$ is now nonsingular.

The basic counting process methodology developed in Section 3 is itself of considerable power, as is indicated by the variety of examples covered. Nevertheless, its applicability can be extended to many more complicated counting situations, in particular by use of some sojourn time information or by augmentation of the state space (simple examples of which are given in Section 5), or by using appropriate marked point processes to determine joint properties of two or more counting processes defined on the same underlying process. We envisage that there will be numerous other applications in a wide range of settings.

\section{Acknowledgements}

This work was supported by grants GR/J98356 and GR/R97368/01 from the UK Engineering and Physical Sciences Research Council. We thank Professor Jeffrey J. Hunter for his interest in this work, Dr Assad Jalali for sharing some related work of his own, and the referees for their helpful comments.

\section{References}

Asmussen, S. (2003). Applied Probability and Queues (Appl. Math. 51), 2nd edn. Springer, New York.

BALL, F. G. (1997). Empirical clustering of bursts of openings in Markov and semi-Markov models of single channel gating incorporating time interval omission. Adv. Appl. Prob. 29, 909-946.

Ball, F. G. AND Davies, S. S. (1997). Clustering of bursts of openings in Markov and semi-Markov models of single channel gating. Adv. Appl. Prob. 29, 92-113.

BALl, F. G. AND SANSOM, M. S. P. (1987). Temporal clustering of ion channel openings incorporating time interval omission. IMA J. Math. Appl. Med. Biol. 4, 333-361.

Ball, F. G., Milne, R. K. And Yeo, G. F. (1993). On the exact distribution of observed open times in single ion channel models. J. Appl. Prob. 30, 529-537.

Ball, F. G., Milne, R. K. and Yeo, G. F. (1994). Continuous-time Markov chains in a random environment, with applications to ion channel modelling. Adv. Appl. Prob. 26, 919-946.

Bellman, R. (1970). Introduction to Matrix Analysis, 2nd edn. McGraw-Hill, New York.

Çinlar, E. (1969). Markov renewal theory. Adv. Appl. Prob. 1, 123-187.

ÇInlar, E. (1975a). Introduction to Stochastic Processes. Prentice-Hall, Englewood Cliffs, NJ. 
ÇInlaR, E. (1975b). Markov renewal theory: a survey. Manag. Sci. 31, 727-752.

Colquhoun, D. and Hawkes, A. G. (1982). On the stochastic properties of bursts of single ion channel openings and of clusters of bursts. Phil. Trans. R. Soc. London B 300, 1-59.

CsENKI, A. (1991). Some renewal-theoretic investigations in the theory of sojourn times in finite Markov processes. J. Appl. Prob. 28, 822-832.

Csenki, A. (1994). Dependability for Systems with a Partitioned State Space. Markov and Semi-Markov Theory and Computational Implementation (Lecture Notes Statist. 90). Springer, New York.

CSENKI, A. (1995). The number of visits to a subset of the state space by a discrete-parameter semi-Markov process. Statist. Prob. Lett. 22, 71-77.

Daley, D. J. And Vere-Jones, D. (2003). An Introduction to the Theory of Point Processes, Vol 1, Elementary Theory and Methods, 2nd edn. Springer, New York.

DARRoCH, J. N. AND MoRrIS, K. W. (1967). Some passage-time generating functions for discrete-time and continuoustime finite Markov chains. J. Appl. Prob. 4, 496-507.

Hawkes, A. G., Jalali, A. and Colquhoun, D. (1990). The distributions of the apparent open times and shut times in a single channel record when brief events cannot be detected. Phil. Trans. R. Soc. London A 332, 511-538.

Hunter, J. J. (1969). On the moments of Markov renewal processes. Adv. Appl. Prob. 1, 188-210.

KeILson, J. (1969). On the matrix renewal function for Markov renewal processes. Ann. Math. Statist. 40, $1901-1907$.

KeIlson, J. (1979). Markov Chain Models—Rarity and Exponentiality. Springer, New York.

Kemeny, J. G. And Snell, J. L. (1976). Finite Markov Chains. Springer, New York.

Masuda, Y. AND Sumita, U. (1987). Analysis of a counting process associated with a semi-Markov process: number of entries into a subset of the state space. Adv. Appl. Prob. 19, 767-783.

Neuts, M. (1995). Algorithmic Probability: a Collection of Problems. Chapman and Hall, London.

ZHENG, Q. (2001). On the dispersion index of a Markovian molecular clock. Math. Biosci. 172, 115-128. 